Preprints of the

Max Planck Institute for

Research on Collective Goods

Bonn 2011/8

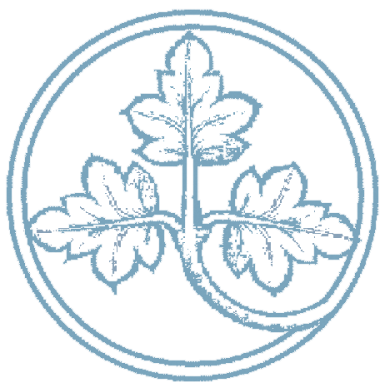

Incomplete-Information

Models of Large Economies with Anonymity:

Existence and Uniqueness of Common Priors

Martin Hellwig

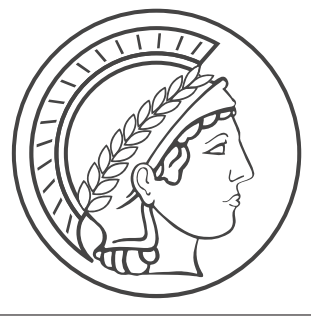




\section{Incomplete-Information Models of Large Economies with Anonymity: Existence and Uniqueness of Common Priors}

Martin Hellwig

May 2011 


\title{
Incomplete-Information Models of Large Economies with Anonymity: Existence and Uniqueness of Common Priors*
}

\author{
Martin F. Hellwig \\ Max Planck Institute for Research on Collective Goods \\ Kurt-Schumacher-Str. 10, D- 53113 Bonn, Germany \\ hellwig@coll.mpg.de
}

May 10, 2011

\begin{abstract}
The paper provides a specification of belief systems for models of large economies with anonymity in which aggregate states depend only on crosssection distributions of types. For belief systems satisfying certain conditions of mutual absolute continuity, the paper gives a necessary and sufficient condition for the existence of a common prior. Under the given conditions, the common prior is unique.

Key Words: Belief systems, consistency, common prior, large economy.

JEL: C70, D82, D83.
\end{abstract}

\section{Incomplete Information in a Large Economy with Anonymity}

In this paper, I consider the existence and uniqueness of common priors in largeeconomy models with incomplete information. A large economy is represented by an atomless measure space of agents, $(A, \mathcal{A}, \alpha)$. Incomplete information is modelled by means of an abstract type space $[(T, \mathcal{T}), t, \theta, \beta]$, where $T$, the space of potential "types", is a complete separable metrice space, $\mathcal{T}$ is the Borel $\sigma$ algebra on $T, t: A \rightarrow T$ is a measurable mapping that assigns types to agents, $\theta: T \rightarrow \Theta$ is a measurable mapping that specifies an agent's payoff parameters or, more generally, his economic characteristics as a function of his type, and $\beta: T \rightarrow \mathcal{M}(\mathcal{M}(T))$ is a measurable mapping that specifies an agent's beliefs as a function of his type. For any $t$, the belief $\beta(t)$ is a probability distribution over cross-section distributions of types in the economy. Thus, $\beta(t) \in \mathcal{M}(\mathcal{M}(T))$.

*For very helpful discussions and comments, I am grateful to Felix Bierbrauer, Christoph Engel, Alia Gizatulina, and Christian Hellwig. 
This formulation of incomplete information departs from the personalistic approach where each agent is assumed to receive a signal about the underlying state of the world and uses the signal to form probabilistic beliefs about the state of the world, about the signals received by the other agents and about the other agents' beliefs that are induced by their signals (Harsanyi [5], Aumann [1], Mertens and Zamir [9]). In the formulation here, agents do not form beliefs about any particular other agents. They only form beliefs about the crosssection distribution of the other agents' characteristics. The idea is that, in some sense, all agents are alike and "the system" satisfies an anonymity condition by which the outcome for any one agent depends only on his own characteristics and on the cross-section distribution of characteristics of agents. In the large economy, of course, the cross-section distribution of characteristics is unaffected by the characteristics of any one individual.

For an application, the reader is referred to the analysis of public-good provision in a large economy by Bierbrauer and Hellwig [2]. In that paper, the payoff parameter $\theta(t(a))$ is an indicator of the benefit that agent $a$ obtains from the public good. An anonymous social choice function determines the level of public-good provision as a function of the cross-section distribution of publicgood preferences without taking account of which agents value the public good highly and which ones do not. For instance, the social choice function might stipulate that the public good be provided if and only if the cross-section mean benefit $\int \theta(t(a)) d \alpha(a)$ exceeds the per capita cost of provision. Or it might stipulation that the public good be provided if and only if there is a sufficiently high share of agents for whom the benefit $\theta(t(a))$ exceeds the per capita provision cost. ${ }^{1}$ A mechanism that implements such a social choice function will condition outcomes on the cross-section distribution of the messages that it receives, without taking account of which agent is sending which message.

For the individual agent who is thinking about what the other agents are doing, it is therefore enough to think about the cross-section distribution of messages that the other agents are sending to "the system". In terms of an abstract type space formulation, this is equivalent to his forming expectations about the cross-section distribution of types and about the dependence of messages on types. His expectations about the cross-section distribution of types are represented by his belief $\beta(t(a)) \in \mathcal{M}(\mathcal{M}(T))$. His expectations about the dependence of reports on types are given by the specification of a reporting strategy $r(\cdot)$ as a candidate for a Bayes-Nash equilibrium of the strategic game that is defined by the mechanism implementing the social choice function.

In the large-economy model, as in the finite-agent models, the question arises under what conditions the beliefs $\beta(t)$ can be interpreted as a result of conditioning on $t$ in a stochastic model with a common prior. I study this question for stochastic models in which the assignments of types to agents satisfy a conditional law of large numbers. The cross-section distribution of types $\delta$ is treated as the realization of a random variable $\tilde{\delta}$, which is defined on some probability

\footnotetext{
${ }^{1}$ The main result of Bierbrauer and Hellwig (2010) shows that if the social choice function is to be robustly implementable and coalition-proof, the social choioce function can only condition on population share of proponents and opponents of provision.
} 
space $(\Omega, \mathcal{F}, \nu)$ and which takes values in $\mathcal{M}(T)$. For each $a \in A$, the type $t(a)$ of agent $a$ is also treated as the realization of a random variable $\tilde{t}(\cdot, a)$ that is defined on $(\Omega, \mathcal{F}, \nu)$; the conditional distribution of this random variable given the event $\tilde{\delta}=\delta$ is assumed to be just equal to $\delta$. The joint dependence of the realizations $\tilde{t}(\omega, a)$ of agents' types on the underlying state $\omega$ is such that, for almost every $\omega \in \Omega$, the cross-section distribution of types well defined and equal to $\tilde{\delta}(\omega)$.

In this specification, the random variable $\tilde{\delta}$ plays a dual role. For each agent, $\tilde{\delta}$ is the conditional distribution of the random variable $\tilde{t}(\cdot, a)$ that determines the agent's type. For the economy as a whole, $\tilde{\delta}$ is the cross-section distribution of types. The two roles of $\tilde{\delta}$ are compatible with each other if the random variables $\tilde{t}(\cdot, a), a \in A$, satisfy a conditional law of large numbers. ${ }^{2}$

In the given specification, the random variable $\tilde{\delta}$ has the probability distribution $Q=\nu \circ \tilde{\delta}^{-1}$. Because the conditional distribution of $\tilde{t}(\cdot, a)$ given $\tilde{\delta}$ is just $\tilde{\delta}$, it follows that the joint distribution of $\tilde{t}(\cdot, a)$ and $\tilde{\delta}$ is given by a measure $\Phi \in \mathcal{M}(T \times \mathcal{M}(T))$ such that

$$
\Phi\left(B_{t} \times B_{\delta}\right)=\int_{B_{\delta}} \delta\left(B_{t}\right) Q(d \delta)
$$

for all measurable sets $B_{t} \subset T$ and $B_{\delta} \subset \mathcal{M}(T)$. I will refer to $\Phi$ as a prior. The belief system $\beta: T \rightarrow \mathcal{M}(\mathcal{M}(T))$ is compatible with the prior $\Phi$ if the map $\tilde{t} \rightarrow \beta(\tilde{t})$ is a regular conditional distribution for $\tilde{\delta}$ given $\tilde{t}$, i.e. if

$$
\Phi\left(B_{t} \times B_{\delta}\right)=\int_{B_{1}} \beta\left(B_{\delta} \mid t\right) \Phi(d t \times \mathcal{M}(T))
$$

for all measurable sets $B_{t} \subset T$ and $B_{\delta} \subset \mathcal{M}(T)$. In this case, I will say that $\Phi$ is a common prior for the belief system $\beta$.

In the following section, I will give conditions on the belief system under which a common prior exists and is unique. The concluding section discusses the role of the assumptions that are used to ensure the desired result.

\section{Existence and Uniqueness of a Common Prior}

The problem of existence and uniqueness of a common prior in a large-economy model with anonymity is formally equivalent to the problem of existence and uniqueness of a common prior in a certain two-player model. In this equivalent two-player model, player 1 has the type space $T$ and player 2 the type space $\mathcal{M}(T)$. Belief systems are given by the functions $t \rightarrow \beta(t)$ and $\delta \rightarrow \delta$. For each

\footnotetext{
${ }^{2}$ Beginning with Judd [8] and Feldman and Gilles [4], there is an extensive literature on the law of large numbers for large economies. Sun [15] provides a formulation in which an assumption of essential pairwise independence yields a law of large numbers on any nonnegligible subset of agents; see also Sun and Zhang [16] and Podczeck [11]. For the conditional law of law numbers that is presumed here, the formulation of Sun [15] must be extended so as to allow for conditional, as opposed to overall independence.
} 
$t \in T$, the probabilistic beliefs of player 1 about the type of player 2 are given by $\beta(t) \in \mathcal{M}(\mathcal{M}(T))$; for each $\delta \in \mathcal{M}(T)$, the probabilistic beliefs of player 2 about the type of player 1 are given by $\delta$. A measure $\Phi \in \mathcal{M}(T \times \mathcal{M}(T))$ is a common prior for the belief system $\beta$ in the large-economy model if and only if it is also a common prior for the equivalent two-player model.

Existence and uniqueness of common priors in finite-player models have mostly been studied under the assumption that type sets are finite. ${ }^{3}$ Under this assumption, one easily obtains:

Proposition 1 Assume that $T$ is a finite set. Assume also that there exists a finite set $D \subset \mathcal{M}(T)$ such that $\beta(t) \in \mathcal{M}(D)$ for all $t \in T$. If $\beta(\{\delta\} \mid t)>0$ and $\delta(\{t\})>0$ for all $t \in T$ and all $\delta \in D$, a common prior for the belief system $\beta$ exists if and only if

$\delta_{1}\left(\left\{t_{1}\right\}\right) \cdot \beta\left(\left\{\delta_{1}\right\} \mid t_{2}\right) \cdot \delta_{2}\left(\left(\left\{t_{2}\right\}\right) \cdot \beta\left(\left\{\delta_{2}\right\} \mid t_{1}\right)=\beta\left(\left\{\delta_{1}\right\} \mid t_{1}\right) \cdot \delta_{2}\left(\left\{t_{1}\right\}\right) \cdot \beta\left(\left\{\delta_{2}\right\} \mid t_{2}\right) \cdot \delta_{1}\left(\left\{t_{2}\right\}\right)\right.$

for all $t_{1}, t_{2}$ in $T$ and all $\delta_{1}, \delta_{2}$ in $D$. The common prior is unique.

The "only if" part of this proposition and the uniqueness claim follow from the argument used in the proof of Lemma 2, p. 490, and Theorem III, p. 488, in Harsanyi [5]. The "if" part of the proposition follows from Hellwig's [7] addendum to the result of Rodrigues-Neto [12]. ${ }^{4}$ The proposition actually is a special case of the more general Proposition 4 below.

For a large-economy model where beliefs are defined as probability distributions over cross-section distributions of types, the assumption of a finite number of possible "types" is unduly restrictive. Under this assumption, cross-section distributions of characteristics must assign positive weights to the individual elements of $T$. There is no room for the notion that the cross-section distributions of characteristics might be dispersed.

In applications, however, dispersedness of cross-section distributions is a useful property to have. With this property, there is a presumption that, for any two arbitrarily specified alternatives that people might choose from, the set of agents who are indifferent between these alternatives is a null set and can be neglected. In Bierbrauer and Hellwig [2], for instance, dispersedness of the cross-section distribution of public-good preferences implies that, in voting on whether the public good is to be provided or not, there is no need to take account of abstentions.

Given these concerns, the following analysis generalizes Proposition 1 so as to allow for uncountable type sets and for atomless cross-section distributions. Before proceeding with the analysis itself, I note that, even with finite type sets, Proposition 1 is not fully general, but is restricted to belief systems satisfying

\footnotetext{
${ }^{3}$ Feinberg [3] uses a syntactic approach to characterize common priors. Among the various semantic approaches, Morris [10] and Samet [14] rely on separation arguments, Samet [13] on the analysis of Markov processes that can be associated with the given belief system, Rodrigues-Neto [12] and Hellman and Samet [6] on the verification of the so-called cycle equations that are associated with the given belief system.

${ }^{4}$ See also Hellman and Samet $[6]$.
} 
$\beta(\{\delta\} \mid t)>0$ and $\delta(\{t\})>0$ for all $t \in T$ and all $\delta \in D$. Without this restriction, the set of conditions that are necessary and sufficient for the existence and uniqueness of a common prior would be significantly larger and the equations are more complex. ${ }^{5}$ To avoid this complexity even as I turn from the finite-type case to the general case, I will restrict the analysis to belief systems having the following properties:

Property 1 The beliefs $\beta(t) \in \mathcal{M}(\mathcal{M}(T))$ are mutually absolutely continuous, i.e., for any $t$ and $t^{\prime}$ in $T$ and any measurable set $B_{\delta} \subset \mathcal{M}(T), \beta\left(B_{\delta} \mid t\right)=0$ if and only if $\beta\left(B_{\delta} \mid t^{\prime}\right)=0$.

Property 2 The common support $D$ of the measures $\beta(t), t \in T$, has the property that the measures $\delta \in D$ are mutually absolutely continuous, i.e., for any $\delta$ and $\delta^{\prime}$ in $D$ and any measurable set $B_{t} \subset T, \delta\left(B_{t}\right)=0$ if and only if $\delta^{\prime}\left(B_{t}\right)=0$.

For finite $T$ and $D$, these properties are equivalent to the positivity assumptions about $\beta(\{\delta\} \mid t)$ and $\delta(\{t\})$ in Proposition 1. By the Radon-Nikodym Theorem, they are equivalent to the following properties:

Property $1^{*}$ There exists a measurable function $f: \mathcal{M}(T) \times T \times T \rightarrow \mathbb{R}_{+}$ such that, for any $t$ and $t^{\prime}$ in $T$ and any measurable set $B_{\delta} \subset \mathcal{M}(T)$,

$$
\beta\left(B_{\delta} \mid t^{\prime}\right)=\int_{B_{\delta}} f\left(\delta, t^{\prime}, t\right) \beta(d \delta \mid t) .
$$

Property $2^{*}$ The common support $D$ of the measures $\beta(t)$ for $t \in T$ has the property that there exists a measurable function $g: T \times D \times D$ such that, for any $\delta$ and $\delta^{\prime}$ in $D$ and any measurable set $B_{t} \subset T$,

$$
\delta^{\prime}\left(B_{t}\right)=\int_{B_{t}} g\left(t, \delta^{\prime}, \delta\right) \delta(d t) .
$$

The following implications of Properties $1^{*}$ and $2^{*}$ will be useful in the subsequent analysis.

Lemma 2 The function $f$ in Property $1^{*}$ satisfies the equation

$$
f\left(\delta, t^{\prime \prime}, t\right)=f\left(\delta, t^{\prime \prime}, t^{\prime}\right) \cdot f\left(\delta, t^{\prime}, t\right)
$$

for $\beta(t)$-almost all $\delta \in \mathcal{M}(T)$, for all $t, t^{\prime}, t^{\prime \prime}$ in $T$. The function $g$ in Property $2^{*}$ satisfies the equation

$$
g\left(t, \delta^{\prime \prime}, \delta\right)=g\left(t, \delta^{\prime \prime}, \delta^{\prime}\right) \cdot g\left(t, \delta^{\prime}, \delta\right)
$$

for $\delta$-almost all $t \in T$, for all $\delta, \delta^{\prime}, \delta^{\prime \prime}$ in $D$.

\footnotetext{
${ }^{5}$ To establish the existence of a common prior, the full set of "cycle equations" defined in Rodrigues-Neto [12] must be verified. In contrast, the "if" part of Proposition 1 rests on the finding in [7] that, for belief systems satisfying $\beta(\{\delta\} \mid t)>0$ and $\delta(\{t\})>0$ for all $t \in T$ and all $\delta \in D$, it suffices to consider these equations for "cycles" of length four or less because the equations for longer cycles follow automatically. Without positivity, this is not necessarily true.
} 
Proof. For any measurable set $B_{\delta} \subset \mathcal{M}(T)$ and any $t, t^{\prime}, t^{\prime \prime}$, Property $1^{*}$ implies

$$
\beta\left(B_{\delta} \mid t^{\prime \prime}\right)=\int_{B_{\delta}} f\left(\delta, t^{\prime \prime}, t^{\prime}\right) \beta\left(d \delta \mid t^{\prime}\right)=\int_{B_{\delta}} f\left(\delta, t^{\prime \prime}, t^{\prime}\right) \cdot f\left(\delta, t^{\prime}, t\right) \beta(d \delta \mid t),
$$

so that the product $f\left(\cdot, t^{\prime \prime}, t^{\prime}\right) \cdot f\left(\cdot, t^{\prime}, t\right)$ is a Radon-Nikodym derivative of the measure $\beta\left(t^{\prime \prime}\right)$ with respect to the measure $\beta(t)$. The first statement follows because the Radon-Nikodym derivative of one measure with respect to another is unique up to a set of measure zero. The second statement follows by a parallel argument.

Lemma 3 The functions $f$ and $g$ in Properties $1^{*}$ and $2^{*}$ satisfy

$$
f\left(\delta, t, t^{\prime}\right)>0
$$

and

$$
g\left(t, \delta, \delta^{\prime}\right)>0
$$

for $\delta^{\prime} \times \beta\left(t^{\prime}\right)$-almost all $(t, \delta) \in T \times D$, for all $t^{\prime} \in T$ and all $\delta^{\prime} \in D$.

Proof. Fix $t^{\prime} \in T$ and $\delta^{\prime} \in D$. Upon setting $t^{\prime \prime}=t$ in the first statement of Lemma 2, one finds that

$$
f\left(\delta, t, t^{\prime}\right) \cdot f\left(\delta, t^{\prime}, t\right)=1
$$

for $\beta(t)$-almost all $\delta \in \mathcal{M}(T)$, for all $t$ and $t^{\prime}$ in $T$. This is only possible if $f\left(\delta, t, t^{\prime}\right)>0$ for $\beta(t)$-almost all $\delta \in \mathcal{M}(T)$, for all $t$ and $t^{\prime}$ in $T$. Because the measures $\beta(t)$ and $\beta\left(t^{\prime}\right)$ are mutually absolutely continuous, it follows that $f\left(\delta, t, t^{\prime}\right)>0$ for $\beta\left(t^{\prime}\right)$-almost all $\delta \in \mathcal{M}(T)$, for all $t$ and $t^{\prime}$ in $T$. Thus, if $h$ is the characteristic function of the set

$$
\left\{(t, \delta) \mid f\left(\delta, t, t^{\prime}\right)=0\right\}
$$

we must have

$$
\int_{T} \int_{D} h(t, \delta) \beta\left(d \delta \mid t^{\prime}\right) \delta^{\prime}(d t)=\int_{D} \int_{T} h(t, \delta) \delta^{\prime}(d t) \beta\left(d \delta \mid t^{\prime}\right)=0
$$

hence $h(t, \delta)=0$ and $f\left(\delta, t, t^{\prime}\right)>0$ for $\delta^{\prime} \times \beta\left(t^{\prime}\right)$-almost all $(t, \delta) \in T \times D$. The second statement follows by a parallel argument.

Lemma 3 provides for an explicit generalization of the positivity assumptions about $\beta(\{\delta\} \mid t)$ and $\delta(\{t\})$ in Proposition 1. This provides the basis for the following generalization of Proposition 1, the main result of this paper.

Proposition 4 Assume that the type space $T$ and the belief system $\beta: T \rightarrow$ $\mathcal{M}(\mathcal{M}(T))$ have Properties 1 and 2, and let $f$ and $g$ be the associated density 
functions. Then, a common prior for the belief system $\beta$ exists if and only if, for any $t_{0} \in T$ and $\delta_{0} \in D$, the equation

$$
\begin{aligned}
& g\left(t_{1}, \delta_{1}, \delta_{0}\right) \cdot f\left(\delta_{1}, t_{2}, t_{0}\right) \cdot g\left(t_{2}, \delta_{2}, \delta_{0}\right) \cdot f\left(\delta_{2}, t_{1}, t_{0}\right) \\
= & f\left(\delta_{1}, t_{1}, t_{0}\right) \cdot g\left(t_{1}, \delta_{2}, \delta_{0}\right) \cdot f\left(\delta_{2}, t_{2}, t_{0}\right) \cdot g\left(t_{2}, \delta_{1}, \delta_{0}\right)
\end{aligned}
$$

holds for $\delta_{0}$-almost all $t_{1}$ and $t_{2}$ in $T$ and $\beta\left(t_{0}\right)$-almost all $\delta_{1}$ and $\delta_{2}$ in $D$. The common prior is unique.

Proof. Suppose that $\Phi \in \mathcal{M}(T \times \mathcal{M}(T))$ is a common prior for the belief system $\beta$. Let $\Phi_{t} \in \mathcal{M}(T)$ be the induced marginal distribution on $T$ and $\Phi_{\delta} \in \mathcal{M}(\mathcal{M}(T))$ the induced marginal distribution on $\mathcal{M}(T)$. Thus, for any $B_{t} \subset T$ and $B_{\delta} \subset D$,

$$
\Phi\left(B_{t} \times B_{\delta}\right)=\int_{B_{\delta}} \delta\left(B_{t}\right) \Phi_{\delta}(d \delta)=\int_{B_{t}} \beta\left(B_{\delta} \mid t\right) \Phi_{t}(d t) .
$$

I claim that the measure $\Phi_{t}$ and the measures $\delta \in D$ are mutually absolutely continuous. To see this, note that, for $B_{\delta}=D$, equation (12) implies

$$
\Phi_{t}\left(B_{t}\right)=\int_{D} \delta\left(B_{t}\right) \Phi_{\delta}(d \delta)
$$

By Property $2^{*}$ and Fubini's theorem, it follows that

$$
\begin{aligned}
\Phi_{t}\left(B_{t}\right) & =\int_{D} \int_{B_{t}} g\left(t, \delta, \delta_{0}\right) \delta_{0}(d t) \Phi_{\delta}(d \delta) \\
& =\int_{B_{t}} \int_{D} g\left(t, \delta, \delta_{0}\right) \Phi_{\delta}(d \delta) \delta_{0}(d t)
\end{aligned}
$$

for any $\delta_{0} \in D$. (14) immediately implies that $\Phi_{t}$ is absolutely continuous with respect to $\delta_{0}$. By Lemma 3, (14) also implies that $\delta_{0}$ is absolutely continuous with respect to $\Phi_{t}$.

By an analogous argument, the measure $\Phi_{\delta}$ and the measures $\beta(t), t \in T$ are also mutually absolutely continuous: For $B_{t}=T$, (12) implies

$$
\begin{aligned}
\Phi_{\delta}\left(B_{\delta}\right) & =\int_{T} \beta\left(B_{\delta} \mid t\right) \Phi_{t}(d t) \\
& =\int_{T} \int_{B_{\delta}} f\left(\delta, t, t_{0}\right) \beta\left(d \delta \mid t_{0}\right) \Phi_{t}(d t) \\
& =\int_{B_{\delta}} \int_{T} f\left(\delta, t, t_{0}\right) \Phi_{t}(d t) \beta\left(d \delta \mid t_{0}\right),
\end{aligned}
$$

so that, again, absolute continuity of $\Phi_{\delta}$ with respect to $\beta\left(t_{0}\right)$ is immediate, and absolute continuity of $\beta\left(t_{0}\right)$ with respect to $\Phi_{\delta}$ follows from Lemma 3 .

Equations (14) and (15) also show that the Radon-Nikodym derivatives of $\Phi_{\delta}$ and $\Phi_{\delta}$ with respect to $\beta\left(t_{0}\right)$ and $\delta_{0}$ are given by the functions

$$
\varphi_{t}\left(\cdot \mid \delta_{0}\right):=\int_{D} g\left(\cdot, \delta, \delta_{0}\right) \Phi_{\delta}(d \delta)
$$


and

$$
\varphi_{\delta}\left(\cdot \mid t_{0}\right):=\int_{T} f\left(\cdot, t, t_{0}\right) \Phi_{t}(d t) .
$$

Given these findings, equation (12) can be rewritten in the form

$$
\Phi\left(B_{t} \times B_{\delta}\right)=\int_{B_{\delta}} \int_{B_{t}} g\left(t, \delta, \delta_{0}\right) \varphi_{\delta}\left(\delta, t_{0}\right) \delta_{0}(d t) \beta\left(d \delta \mid t_{0}\right)
$$

and

$$
\Phi\left(B_{t} \times B_{\delta}\right)=\int_{B_{t}} \int_{B_{\delta}} f\left(\delta, t, t_{0}\right) \varphi_{t}\left(t, \delta_{0}\right) \beta\left(d \delta \mid t_{0}\right) \delta_{0}(d t) .
$$

Thus, for any $t_{0} \in T$ and $\delta_{0} \in D$, the common prior $\Phi$ is absolutely continuous with respect to the product measure $\beta\left(t_{0}\right) \times \delta_{0}$, with a density $\varphi\left(\cdot, \cdot \mid t_{0}, \delta_{0}\right)$ satisfying

$$
\varphi\left(t, \delta \mid t_{0}, \delta_{0}\right)=g\left(t, \delta, \delta_{0}\right) \varphi_{\delta}\left(\delta, t_{0}\right)=f\left(\delta, t, t_{0}\right) \varphi_{t}\left(t, \delta_{0}\right)
$$

for $\delta_{0}$-almost all $t \in T$ and $\beta\left(t_{0}\right)$-almost all $\delta \in D$.

Consider the second equation in (20) with $t$ and $\delta$ replaced by different constellations of $t_{1}, t_{2} \in T$ and $\delta_{1}, \delta_{2} \in D$. This yields the equation

$$
\begin{aligned}
& g\left(t_{1}, \delta_{1}, \delta_{0}\right) \varphi_{\delta}\left(\delta_{1}, t_{0}\right) \cdot f\left(\delta_{1}, t_{2}, t_{0}\right) \varphi_{t}\left(t_{2}, \delta_{0}\right) \\
& \cdot g\left(t_{2}, \delta_{2}, \delta_{0}\right) \varphi_{\delta}\left(\delta_{2}, t_{0}\right) \cdot f\left(\delta_{2}, t_{1}, t_{0}\right) \varphi_{t}\left(t_{1}, \delta_{0}\right) \\
= & f\left(\delta_{1}, t_{1}, t_{0}\right) \varphi_{t}\left(t_{1}, \delta_{0}\right) \cdot g\left(t_{1}, \delta_{2}, \delta_{0}\right) \varphi_{\delta}\left(\delta_{2}, t_{0}\right) \\
& \cdot f\left(\delta_{2}, t_{2}, t_{0}\right) \varphi_{t}\left(t_{2}, \delta_{0}\right) \cdot g\left(t_{2}, \delta_{1}, \delta_{0}\right) \varphi_{\delta}\left(\delta_{1}, t_{0}\right) .
\end{aligned}
$$

Because the measures $\Phi_{t}$ and $\delta_{0}$, as well as the measures $\Phi_{\delta}$ and $\beta\left(t_{0}\right)$, are mutually absolutely continuous, the same argument as in Lemma 3 implies that

$$
\varphi_{t}\left(t, \delta_{0}\right)>0 \text { for } \delta_{0} \text {-almost all } t
$$

and

$$
\varphi_{\delta}\left(\delta, t_{0}\right)>0 \text { for } \beta\left(t_{0}\right) \text {-almost all } \delta .
$$

For $\delta_{0}$-almost all $t_{1}$ and $t_{2}$ in $T$ and $\beta\left(t_{0}\right)$-almost all $\delta_{1}$ and $\delta_{2}$ in $D$, therefore, the terms $\varphi_{\delta}\left(\delta_{1}, t_{0}\right), \varphi_{t}\left(t_{2}, \delta_{0}\right), \varphi_{\delta}\left(\delta_{2}, t_{0}\right)$, and $\varphi_{t}\left(t_{1}, \delta_{0}\right)$ in $(21)$ can be divided out. This yields (11).

To prove the "if" part of the proposition, suppose that, for some $t_{0} \in T$ and $\delta_{0} \in D$, equation (11) is satisfied for $\delta_{0}$-almost all $t_{1}$ and $t_{2}$ in $T$ and $\beta\left(t_{0}\right)$-almost all $\delta_{1}$ and $\delta_{2}$ in $D$. By Lemma 3 , there exist some $t_{1}$ and $\delta_{1}$ so that

$$
f\left(\delta_{1}, t_{1}, t_{0}\right)>0, \quad g\left(t_{1}, \delta_{1}, \delta_{0}\right)>0,
$$

and

$$
g\left(t_{1}, \delta, \delta_{0}\right)>0 \text { for } \beta\left(t_{0}\right) \text {-almost all } \delta .
$$


For any $t$ and $\delta$, define

$$
\begin{aligned}
\varphi\left(t, \delta, t_{0}, \delta_{0}\right)= & \lambda\left(t_{0}, \delta_{0}\right) \cdot \frac{f\left(\delta, t_{1}, t_{0}\right)}{f\left(\delta_{1}, t_{1}, t_{0}\right)} \cdot \frac{g\left(t, \delta, \delta_{0}\right)}{g\left(t_{1}, \delta, \delta_{0}\right)} \\
& \text { if } g\left(t_{1}, \delta, \delta_{0}\right)>0 \text { and } \\
\varphi\left(t, \delta, t_{0}, \delta_{0}\right)= & 0 \text { if } g\left(t_{1}, \delta, \delta_{0}\right)=0,
\end{aligned}
$$

where

$$
\lambda\left(t_{0}, \delta_{0}\right):=\left[\int_{D} \int_{T} \frac{f\left(\delta, t_{1}, t_{0}\right)}{f\left(\delta_{1}, t_{1}, t_{0}\right)} \cdot \frac{g\left(t, \delta, \delta_{0}\right)}{g\left(t_{1}, \delta, \delta_{0}\right)} \delta_{0}(d t) \beta\left(d \delta \mid t_{0}\right)\right]^{-1} .
$$

Define a measure $\Phi \in \mathcal{M}(T \times D)$ by setting

$$
\Phi\left(B_{t} \times B_{\delta}\right):=\int_{B_{\delta}} \int_{B_{t}} \varphi\left(t, \delta, t_{0}, \delta_{0}\right) \delta_{0}(d t) \beta\left(d \delta \mid t_{0}\right)
$$

for any measurable $B_{t} \subset T$ and $B_{\delta} \subset D$. Then, by construction,

$$
\begin{aligned}
\Phi\left(B_{t} \times B_{\delta}\right) & =\int_{B_{\delta}} \int_{B_{t}} \lambda \cdot \frac{f\left(\delta, t_{1}, t_{0}\right)}{f\left(\delta_{1}, t_{1}, t_{0}\right)} \cdot \frac{g\left(t, \delta, \delta_{0}\right)}{g\left(t_{1}, \delta, \delta_{0}\right)} \delta_{0}(d t) \beta\left(d \delta \mid t_{0}\right) \\
& =\int_{B_{\delta}} \int_{B_{t}} g\left(t, \delta, \delta_{0}\right) \delta_{0}(d t) \cdot \Phi_{\delta}(d \delta) \\
& =\int_{B_{\delta}} \int_{B_{t}} \delta(d t) \cdot \Phi_{\delta}(d \delta),
\end{aligned}
$$

where $\Phi_{\delta} \in \mathcal{M}(D)$ is given by the formula

$$
\Phi_{\delta}\left(B_{\delta}\right)=\Phi\left(T \times B_{\delta}\right)=\int_{B_{\delta}} \lambda \cdot \frac{f\left(\delta, t_{1}, t_{0}\right)}{f\left(\delta_{1}, t_{1}, t_{0}\right)} \cdot \frac{1}{g\left(t_{1}, \delta, \delta_{0}\right)} \beta\left(d \delta \mid t_{0}\right) .
$$

Equation (25) shows that $\Phi$ satisfies the first equation in (12).

To verify that $\Phi$ also satisfies the second equation in (12), observe that, by Lemma 3 and (11), we also have

$$
\varphi\left(t, \delta, t_{0}, \delta_{0}\right)=\lambda \cdot \frac{f\left(\delta, t, t_{0}\right)}{f\left(\delta_{1}, t, t_{0}\right)} \cdot \frac{g\left(t, \delta_{1}, \delta_{0}\right)}{g\left(t_{1}, \delta_{1}, \delta_{0}\right)}
$$

for $\delta_{0} \times \beta\left(t_{0}\right)$-almost all $(t, \delta)$. Therefore, $(24)$ can be rewritten as

$$
\begin{aligned}
\Phi\left(B_{t} \times B_{\delta}\right) & =\int_{B_{t}} \int_{B_{\delta}} \lambda \cdot \frac{f\left(\delta, t, t_{0}\right)}{f\left(\delta_{1}, t, t_{0}\right)} \cdot \frac{g\left(t, \delta_{1}, \delta_{0}\right)}{g\left(t_{1}, \delta_{1}, \delta_{0}\right)} \delta_{0}(d t) \beta\left(d \delta \mid t_{0}\right) \\
& =\int_{B_{t}} \int_{B_{\delta}} f\left(\delta, t, t_{0}\right) \beta\left(d \delta \mid t_{0}\right) \cdot \Phi_{t}(d t) \\
& =\int_{B_{t}} \int_{B_{\delta}} \beta(d \delta \mid t) \Phi_{t}(d t)
\end{aligned}
$$


where $\Phi_{t} \in \mathcal{M}(T)$ is given by the formula

$$
\Phi_{t}\left(B_{t}\right)=\Phi\left(B_{t} \times D\right)=\int_{B_{t}} \lambda \cdot \frac{1}{f\left(\delta_{1}, t, t_{0}\right)} \cdot \frac{g\left(t, \delta_{1}, \delta_{0}\right)}{g\left(t_{1}, \delta_{1}, \delta_{0}\right)} \delta_{0}(d t) .
$$

Equation (28) implies that $\Phi$ also satisfies the second equation in (12). Thus, $\Phi$ is a common prior for the belief system $\beta$.

To prove uniqueness, I note that, by the argument in the proof of the "only if" part of the proposition, for any $t_{0} \in T$ and $\delta_{0} \in D$, any common prior $\Phi$ for the belief system $\beta$ has a density $\varphi\left(\cdot, \cdot \mid t_{0}, \delta_{0}\right)$ with respect to the measure $\delta_{0} \times \beta\left(t_{0}\right)$ and, moreover, this density satisfies (20). Thus,

$$
\frac{\varphi\left(t^{\prime}, \delta \mid t_{0}, \delta_{0}\right)}{\varphi\left(t, \delta \mid t_{0}, \delta_{0}\right)}=\frac{g\left(t^{\prime}, \delta, \delta_{0}\right)}{g\left(t, \delta, \delta_{0}\right)}
$$

and

$$
\frac{\varphi\left(t, \delta^{\prime} \mid t_{0}, \delta_{0}\right)}{\varphi\left(t, \delta \mid t_{0}, \delta_{0}\right)}=\frac{f\left(\delta^{\prime}, t, t_{0}\right)}{f\left(\delta, t, t_{0}\right)}
$$

for any $t, t^{\prime} \in T$ and any $\delta, \delta^{\prime} \in D$ such that $\varphi\left(t, \delta \mid t_{0}, \delta_{0}\right)>0$ (and therefore $g\left(t, \delta, \delta_{0}\right)>0$ and $\left.f\left(\delta, t, t_{0}\right)>0\right)$. Upon combining (30) and (31), one finds that

$$
\frac{\varphi\left(t_{1}, \delta_{1} \mid t_{0}, \delta_{0}\right)}{\varphi\left(t_{2}, \delta_{2} \mid t_{0}, \delta_{0}\right)}=\frac{f\left(\delta_{1}, t_{2}, t_{0}\right)}{f\left(\delta_{2}, t_{2}, t_{0}\right)} \cdot \frac{g\left(t_{1}, \delta_{1}, \delta_{0}\right)}{g\left(t_{2}, \delta_{1}, \delta_{0}\right)}
$$

for all $t_{1}, t_{2} \in T, \delta_{1}, \delta_{2} \in D$ such that $g\left(t_{2}, \delta_{1}, \delta_{0}\right)>0$ and $f\left(\delta_{1}, t_{2}, t_{0}\right)>0$. By Lemma 3 , therefore, for any common prior $\Phi$ and $\delta_{0} \times \beta\left(t_{0}\right)$-almost all pairs $\left(t_{1}, \delta_{1}\right),\left(t_{2}, \delta_{2}\right)$, the ratio of densities $\frac{\varphi\left(t_{1}, \delta_{1} \mid t_{0}, \delta_{0}\right)}{\varphi\left(t_{2}, \delta_{2} \mid t_{0}, \delta_{0}\right)}$ is uniquely determined by the density functions $f$ and $g$ that are given by Properties $1^{*}$ and $2^{*}$. Because the integral of $\varphi\left(\cdot, \cdot \mid t_{0}, \delta_{0}\right)$ with respect to the measure $\delta_{0} \times \beta\left(t_{0}\right)$ must be equal to one, it follows that, up to modifications on a set of $\beta\left(t_{0}\right) \times \delta_{0}$-measure zero, the density function $\varphi\left(\cdot, \cdot \mid t_{0}, \delta_{0}\right)$ itself is uniquely determined by the functions $f$ and $g$. Uniqueness of $\Phi$ follows immediately.

In going through the proposition and its proof, the reader may find it paradoxical that, on the one hand, the common prior is unique and, on the other hand, in the proof of the "if" part of the proposition, the construction of the common prior relies on particular specified pairs $\left(t_{0}, \delta_{0}\right)$ and $\left(t_{1}, \delta_{1}\right)$. The following remarks resolve the paradox by showing that the result of the construction does not in fact depend on the specified $\left(t_{0}, \delta_{0}\right)$ and $\left(t_{1}, \delta_{1}\right)$.

Remark 5 The prior that is defined in (22) - (24) does not depend on the particular pair $\left(t_{0}, \delta_{0}\right)$ that is used in the construction.

Proof. In principle, this remark already follows from the fact that the common prior is unique. However, to clarify the role of mutual absolute continuity, I also 
give a direct argument: By Properties $1^{*}$ and $2^{*}$, the right-hand side of (24) can be written as

$$
\left.\int_{B_{\delta}} \int_{B_{t}} \frac{f\left(\delta, t_{1}, t_{0}\right) \cdot f\left(\delta, t_{0}, t_{0}^{\prime}\right)}{f\left(\delta_{1}, t_{1}, t_{0}\right)} \cdot \frac{g\left(t, \delta, \delta_{0}\right) \cdot g\left(t, \delta_{0}, \delta_{0}^{\prime}\right)}{g\left(t_{1}, \delta, \delta_{0}\right)} \delta_{0}^{\prime}(d t) \beta\left(d \delta \mid t_{0}^{\prime}\right)\right),
$$

where $\left(t_{0}^{\prime}, \delta_{0}^{\prime}\right)$ is any other pair in $T \times D$. By (6) and (7), therefore, (24) can be rewritten as

$$
\begin{aligned}
\Phi\left(B_{t} \times B_{\delta}\right) & \left.=\int_{B_{\delta}} \int_{B_{t}} \lambda^{\prime} \cdot \frac{f\left(\delta, t_{1}, t_{0}^{\prime}\right)}{f\left(\delta_{1}, t_{1}, t_{0}^{\prime}\right)} \cdot \frac{g\left(t, \delta, \delta_{0}^{\prime}\right)}{g\left(t_{1}, \delta, \delta_{0}^{\prime}\right)} \delta_{0}^{\prime}(d t) \beta\left(d \delta \mid t_{0}^{\prime}\right)\right) \\
& \left.=\frac{\lambda^{\prime}}{\lambda\left(t_{0}^{\prime}, \delta_{0}^{\prime}\right)} \int_{B_{\delta}} \int_{B_{t}} \varphi\left(t, \delta, t_{0}^{\prime}, \delta_{0}^{\prime}\right) \delta_{0}^{\prime}(d t) \beta\left(d \delta \mid t_{0}^{\prime}\right)\right) .
\end{aligned}
$$

where $\lambda^{\prime}:=\lambda\left(t_{0}, \delta_{0}\right) \cdot f\left(\delta_{1}, t_{0}, t_{0}^{\prime}\right) \cdot g\left(t_{1}, \delta_{0}, \delta_{0}^{\prime}\right)$ and $\lambda\left(t_{0}^{\prime}, \delta_{0}^{\prime}\right)$ is given by (23) with $\left(t_{0}, \delta_{0}\right)$ replaced by $\left(t_{0}^{\prime}, \delta_{0}^{\prime}\right)$. For $B_{t}=T$ and $B_{\delta}=D$, we have $\Phi\left(B_{t} \times B_{\delta}\right)=1$ and $\left.\int_{B_{\delta}} \int_{B_{t}} \varphi\left(t, \delta, t_{0}^{\prime}, \delta_{0}^{\prime}\right) \delta_{0}^{\prime}(d t) \beta\left(d \delta \mid t_{0}^{\prime}\right)\right)=1$. Therefore, $\lambda^{\prime}=\lambda\left(t_{0}^{\prime}, \delta_{0}^{\prime}\right)$, and (33) can be rewritten as

$$
\left.\Phi\left(B_{t} \times B_{\delta}\right)=\int_{B_{\delta}} \int_{B_{t}} \varphi\left(t, \delta, t_{0}^{\prime}, \delta_{0}^{\prime}\right) \delta_{0}^{\prime}(d t) \beta\left(d \delta \mid t_{0}^{\prime}\right)\right)
$$

for all $B_{t}$ and $B_{\delta}$. Since (34) is just $(24)$ with $\left(t_{0}, \delta_{0}\right)$ replaced by $\left(t_{0}^{\prime}, \delta_{0}^{\prime}\right)$, it follows that $\Phi$ does not depend on the particular pair $\left(t_{0}, \delta_{0}\right)$ that was chosen for the construction.

Remark 6 The prior that is defined in (22) - (24) does not depend on the particular pair $\left(t_{1}, \delta_{1}\right)$ that is used in the construction.

Proof. In principle, this remark also follows from the fact that the common prior is unique. However, to clarify the role of condition (11), I also give a direct argument. Given that the integral of the density $\varphi\left(t, \delta, t_{0}, \delta_{0}\right)$ with respect to the measure $\delta_{0} \times \beta\left(t_{0}\right)$ is equal to one, it suffices to show that the ratio $\varphi\left(t, \delta, t_{0}, \delta_{0}\right) / \varphi\left(t^{\prime}, \delta^{\prime}, t_{0}, \delta_{0}\right)$ is independent of $\left(t_{1}, \delta_{1}\right)$ for $\delta_{0} \times \beta\left(t_{0}\right)$-almost all pairs $(t, \delta),\left(t^{\prime}, \delta^{\prime}\right)$ in $T \times D$. For $\delta_{0} \times \beta\left(t_{0}\right)$-almost all pairs $(t, \delta)$ and $\left(t^{\prime}, \delta^{\prime}\right)$ in $T \times D,(22)$ implies

$$
\frac{\varphi\left(t, \delta, t_{0}, \delta_{0}\right)}{\varphi\left(t^{\prime}, \delta^{\prime}, t_{0}, \delta_{0}\right)}=\frac{f\left(\delta, t_{1}, t_{0}\right) \cdot g\left(t_{1}, \delta^{\prime}, \delta_{0}\right) \cdot g\left(t, \delta, \delta_{0}\right)}{f\left(\delta^{\prime}, t_{1}, t_{0}\right) \cdot g\left(t_{1}, \delta, \delta_{0}\right) \cdot g\left(t^{\prime}, \delta^{\prime}, \delta_{0}\right)} .
$$

Moreover, from (11), with $t_{1}, t^{\prime}, \delta, \delta^{\prime}$ taking the role of $t_{1}, t_{2}, \delta_{1}, \delta_{2}$, one infers that

$$
\begin{aligned}
& f\left(\delta, t_{1}, t_{0}\right) \cdot g\left(t_{1}, \delta^{\prime}, \delta_{0}\right) \cdot f\left(\delta^{\prime}, t^{\prime}, t_{0}\right) \cdot g\left(t^{\prime}, \delta, \delta_{0}\right) \\
= & g\left(t_{1}, \delta, \delta_{0}\right) \cdot f\left(\delta, t^{\prime}, t_{0}\right) \cdot g\left(t^{\prime}, \delta^{\prime}, \delta_{0}\right) \cdot f\left(\delta^{\prime}, t_{1}, t_{0}\right)
\end{aligned}
$$


Thus (35) implies:

$$
\begin{aligned}
\frac{\varphi\left(t, \delta, t_{0}, \delta_{0}\right)}{\varphi\left(t^{\prime}, \delta^{\prime}, t_{0}, \delta_{0}\right)}= & \frac{f\left(\delta, t_{1}, t_{0}\right) \cdot g\left(t_{1}, \delta^{\prime}, \delta_{0}\right) \cdot f\left(\delta^{\prime}, t^{\prime}, t_{0}\right) \cdot g\left(t^{\prime}, \delta, \delta_{0}\right)}{f\left(\delta^{\prime}, t_{1}, t_{0}\right) \cdot g\left(t_{1}, \delta, \delta_{0}\right) \cdot f\left(\delta, t^{\prime}, t_{0}\right) \cdot g\left(t^{\prime}, \delta^{\prime}, \delta_{0}\right)} \\
& \cdot \frac{f\left(\delta, t^{\prime}, t_{0}\right) \cdot g\left(t, \delta, \delta_{0}\right)}{f\left(\delta^{\prime}, t^{\prime}, t_{0}\right) \cdot g\left(t^{\prime}, \delta, \delta_{0}\right)} \\
= & \frac{f\left(\delta, t^{\prime}, t_{0}\right) \cdot g\left(t, \delta, \delta_{0}\right)}{f\left(\delta^{\prime}, t^{\prime}, t_{0}\right) \cdot g\left(t^{\prime}, \delta, \delta_{0}\right)},
\end{aligned}
$$

which is independent of $\left(t_{1}, \delta_{1}\right)$.

Given the formulation of the proposition and its proof, the reader may also wonder about the quantifier for the pair $\left(t_{0}, \delta_{0}\right)$ in Proposition 4 . The first part of the proof shows that, if a common prior exists, then the condition given in the proposition must hold for all $t_{0} \in T$ and $\delta_{0} \in D$. The second part of the proof shows that, if the condition holds for some $t_{0} \in T$ and $\delta_{0} \in D$, then a common prior exists. The apparent incongruity is resolved by:

Remark 7 Equation (11) holds for all $t_{0} \in T$ and $\delta_{0} \in D$ and $\delta_{0}$-almost all $t_{1}, t_{2} \in T$ and $\beta\left(t_{0}\right)$-almost all $\delta_{1}, \delta_{2} \in D$ if and only if it holds for some $t_{0} \in T$ and $\delta_{0} \in D$ and $\delta_{0}$-almost all $t_{1}, t_{2} \in T$ and $\beta\left(t_{0}\right)$-almost all $\delta_{1}, \delta_{2} \in D$.

Proof. As mentioned, the remark is implicit in the proof of the proposition. For a direct proof suppose that the condition of given in the proposition holds for some $t_{0} \in T$ and $\delta_{0} \in D$. Multiply equation (11) by $g\left(t_{1}, \delta_{0}, \delta_{0}^{\prime}\right) \cdot f\left(\delta_{1}, t_{0}, t_{0}^{\prime}\right)$. $g\left(t_{2}, \delta_{0}, \delta_{0}^{\prime}\right) \cdot f\left(\delta_{2}, t_{0}, t_{0}^{\prime}\right)$, for some $t_{0}^{\prime} \in T$ and $\delta_{0}^{\prime} \in D$. By (6) and (7) the resulting equation simplifies to

$$
\begin{aligned}
& g\left(t_{1}, \delta_{1}, \delta_{0}^{\prime}\right) \cdot f\left(\delta_{1}, t_{2}, t_{0}^{\prime}\right) \cdot g\left(t_{2}, \delta_{2}, \delta_{0}^{\prime}\right) \cdot f\left(\delta_{2}, t_{1}, t_{0}^{\prime}\right) \\
= & f\left(\delta_{1}, t_{1}, t_{0}^{\prime}\right) \cdot g\left(t_{1}, \delta_{2}, \delta_{0}^{\prime}\right) \cdot f\left(\delta_{2}, t_{2}, t_{0}^{\prime}\right) \cdot g\left(t_{2}, \delta_{1}, \delta_{0}^{\prime}\right),
\end{aligned}
$$

which is just (11) with $t_{0}$ and $\delta_{0}$ replaced by $t_{0}^{\prime}$ and $\delta_{0}^{\prime}$.

\section{Discussion}

Propositions 1 and 4 involve two sets of conditions, the mutual-absolute-continuity conditions of Properties 1 and 2 and the consistency conditions (11). The mutual-absolute-continuity conditions impose a certain homogeneity on crosssection type distributions and on beliefs:

- All relevant cross-section type distributions assign positive probability to the same sets of types. There is thus no aggregate state under which a relevant individual state can be ruled out.

- All types' beliefs assign positive probability to the same sets of crosssection distributions of types. There is thus no person that "knows" that a given aggregate state cannot occur. 
In the equivalent two-player game, these conditions eliminate the possibility that one player "knows" that the other player "knows" that a certain type of the first player cannot occur. Technically, any element in the information partition intersects every element in the information partition of the other player. ${ }^{6}$ In the large economy, these conditions, i.e., Properties 1 and 2, do not concern the information that one participant may form about another participants' beliefs, but, rather, a limit on the information about the aggregate state that individuals cna infer from the observation of their types. In Bierbrauer and Hellwig [2], this limitation is characterized by saying that the belief system is "relatively uninformative", i.e., the information that a person has does not allow this person rule out any aggregate state that was considered possible ex ante.

The consistency condition (11) has been well known since Harsanyi (1968). Whereas most of the literature discusses this condition in terms of a two-player game with finitely many states, the analysis here involves a large economy with anonymity, with a potentially uncountable set of states. The underlying logic is the same: If (30) and (31) are combined to compute the ratio $\varphi\left(t_{1}, \delta_{1} \mid t_{0}, \delta_{0}\right) / \varphi\left(t_{2}, \delta_{2} \mid t_{0}, \delta_{0}\right)$, the result must independent of whether we compute this ratio from the equation

$$
\frac{\varphi\left(t_{1}, \delta_{1} \mid t_{0}, \delta_{0}\right)}{\varphi\left(t_{2}, \delta_{2} \mid t_{0}, \delta_{0}\right)}=\frac{\varphi\left(t_{2}, \delta_{1} \mid t_{0}, \delta_{0}\right)}{\varphi\left(t_{2}, \delta_{2} \mid t_{0}, \delta_{0}\right)} \cdot \frac{\varphi\left(t_{2}, \delta_{2} \mid t_{0}, \delta_{0}\right)}{\varphi\left(t_{2}, \delta_{1} \mid t_{0}, \delta_{0}\right)},
$$

or from the equation

$$
\frac{\varphi\left(t_{1}, \delta_{1} \mid t_{0}, \delta_{0}\right)}{\varphi\left(t_{2}, \delta_{2} \mid t_{0}, \delta_{0}\right)}=\frac{\varphi\left(t_{1}, \delta_{2} \mid t_{0}, \delta_{0}\right)}{\varphi\left(t_{2}, \delta_{2} \mid t_{0}, \delta_{0}\right)} \cdot \frac{\varphi\left(t_{2}, \delta_{2} \mid t_{0}, \delta_{0}\right)}{\varphi\left(t_{1}, \delta_{2} \mid t_{0}, \delta_{0}\right)} .
$$

When applied to (36), (30) and (31) yield

$$
\frac{\varphi\left(t_{1}, \delta_{1} \mid t_{0}, \delta_{0}\right)}{\varphi\left(t_{2}, \delta_{2} \mid t_{0}, \delta_{0}\right)}=\frac{f\left(\delta_{1}, t_{2}, t_{0}\right)}{f\left(\delta_{2}, t_{2}, t_{0}\right)} \cdot \frac{g\left(t_{1}, \delta_{1}, \delta_{0}\right)}{g\left(t_{2}, \delta_{1}, \delta_{0}\right)},
$$

when applied to (37),

$$
\frac{\varphi\left(t_{1}, \delta_{1} \mid t_{0}, \delta_{0}\right)}{\varphi\left(t_{2}, \delta_{2} \mid t_{0}, \delta_{0}\right)}=\frac{g\left(t_{1}, \delta_{2}, \delta_{0}\right)}{g\left(t_{2}, \delta_{2}, \delta_{0}\right)} \cdot \frac{f\left(\delta_{1}, t_{1}, t_{0}\right)}{f\left(\delta_{2}, t_{1}, t_{0}\right)} .
$$

For these two expressions to be compatible, one needs (11). Because the mutualabsolute-continuity conditions of Properties 1 and 2 ensure that the densities involved are strictly positive almost everywhere, this consistency condition holding for any $t_{1}, t_{2}$ and any $\delta_{1}, \delta_{2}$ is also sufficient for the existence of a common prior when the belief system satisfies Properties 1 and 2 .

\footnotetext{
${ }^{6}$ See Hellwig [7].
} 


\section{References}

[1] Robert Aumann, Agreeing to disagree, Ann. Statist. 4 (1976), 1236-1239.

[2] Felix Bierbrauer and Martin Hellwig, Public-good provision in a large economy, Preprint 2010-02, Max Planck Institute for Research on Collective Goods, Bonn, 2010, http://www.coll.mpg.de/pdf_dat/2010_02online.pdf.

[3] Yossi Feinberg, Characterizing common priors in the form of posteriors, J. Econ. Theory 91 (2000), 127-179.

[4] Mark Feldman and Christian Gilles, An expository note on individual risk without aggregate uncertainty, J. Econ. Theory 35 (1985), 26 - 32.

[5] John C. Harsanyi, Games with incomplete information played by Bayesian players I, II, III, Manag. Sci. 14 (1967), 159-182; Manag. Sci. 14 (1967), 320-334; Manag. Sci. 14 (1968), 486-502.

[6] Ziv Hellman and Dov Samet, How common are common priors?, Discussion Paper \# 532, Center for the Study of Rationality, Hebrew University, Jerusalem, February 2010.

[7] Martin F. Hellwig, From posteriors to priors via cycles: An addendum, Preprint 2011-07, Max Planck Institute for Research on Collective Goods, Bonn, 2011, http://www.coll.mpg.de/pdf_dat/2011_07online.pdf

[8] Kenneth L. Judd, The law of large numbers with a continuum of IID random variables. J. Econ. Theory 35 (1985), 19 - 25.

[9] Jean-Francois Mertens and Shmuel Zamir, Formulation of Bayesian analysis for games with incomplete information, Int. J. Game Theory 14 (1985), 1 -29 .

[10] Stephen Morris, Trade with heterogeneous prior beliefs and asymmetric information, Econometrica 62 (1994), 1326-1347.

[11] Konrad Podczeck, On existence of rich Fubini extensions. Econ. Theory 45 (2010), 1-22.

[12] José Alvaro Rodrigues-Neto, From posteriors to priors via cycles, J. Econ. Theory 144 (2009), 876-883

[13] Dov Samet, Iterative expectations and common priors, Games Econ. Behav. 24 (1998) 131-141.

[14] Dov Samet, Common priors and the separation of convex sets, Games Econ. Behav. 24 (1998), 172-174.

[15] Yeneng Sun, The exact law of large numbers via Fubini extension and characterization of insurable risks, J. Econ. Theory 126 (2006), 31 - 69. 
[16] Yeneng Sun and Yangchao Zhang, Individual risk and Lebesgue extension without aggregate uncertainty, J. Econ. Theory 144 (2009), 432 - 442. 